Tôhoku Math. Journ.

21(1969), 92-101.

\title{
ON TATE'S DUALITY THEOREMS IN GALOIS COHOMOLOGY
}

Dedicated to Professor T.Tannaka on his 60th birthday

\author{
KÔJI UCHIDA
}

(Received August 20, 1968)

1. Notations and Summary of Results. J.Tate [7] has proved duality theorems concerning Galois cohomology groups of finite modules. We generalize some of these theorems to the case of finitely generated modules (See also [6]). Tate has also stated about strict cohomological dimension. Our first aim was to prove this statement which seems to have important meanings in the number theory. But we have not succeeded yet.

Let $G$ be a profinite group. We call $A$ a $G$-module only when it is equal to the union of the submodules $A^{H}$, where $H$ runs over the open subgroups of $G$ and $H$ operates trivially on $A^{H}$. Cohomology groups of such modules in positive dimensions are well known. Cohomology groups in negative and zero dimensions are introduced by Poitou ${ }^{1)}$. By definition

$$
\hat{H}^{i}(G, A)=\underset{\leftarrow}{\lim } \hat{H}^{i}\left(G / H, A^{H}\right), i \leqq 0,
$$

projective limit being taken with respect to deflations when $H$ runs over the open normal subgroups of $G$. We note that they may not be cohomological functors. $\hat{H}^{i}(G, A), i \geqq 1$, denote ordinary cohomology groups. Ordinary cohomology group in dimension zero is denoted as $A^{G}$.

By a global field we mean an algebraic number field of finite degree or an algebraic function field of one variable over a finite constant field. Let $k$ always denote a global field and let $k_{P}$ denote the completion of $k$ with respect to a prime $P$. In the following $G$ always denotes the Galois group of the extension $\Omega / k$. Where $\Omega$ is the maximal algebraic extension of $k$ unramified outside $S$, and $S$ is a non-empty set of primes of $k$ including all the archimedean ones. Then $\hat{H}^{i}(A)$ abbreviates $\hat{H}^{i}(G, A)$. In the local case, let $\hat{H}^{i}\left(k_{P}, A\right)=H^{i}\left(G\left(\bar{k}_{P} / k_{P}\right), A\right)$, and $\hat{H}^{i}\left(o_{P}, A\right)=\hat{H}^{i}(\bar{G}, A)$, when $A$ is a $\bar{G}$-module, where $\bar{G}$ is the Galois group of the maximal unramified extension of $k_{P}$. We also use following notations.

1) After preparing most part of this paper, we knew by [3] that Potiou had adopted this same definition. We do not know his results [5] in detail. 
$N_{G / H}$ : Norm map from $A^{H}$ into $A^{G}$ when. $H$ is an open subgroup of $G$ $N_{G} A=\cap_{H} N_{G / H} A^{H}$ : The universal norms of $A$

$K_{S}$ : The $S$-integers in $K$ which is a finite extension of $k$ contained in $\Omega$

$\Omega_{S}:$ The $S$-integers in $\Omega$

$K_{S}{ }^{\times}, \Omega_{S}{ }^{\times}$: Unit groups of $K_{S}, \Omega_{S}$ respectively

$J_{K}$ : Idele group of $K$

$C_{K}$ : Idele class group of $K$

$J_{S, K}$ : Projection of $J_{K}$ to $S$-components

$C_{S, K}=J_{S, K} / K_{S}$

$U_{K}$ : Subgroup of $J_{K}$ consisting of the ideles whose components are equal to 1 in $S$ and are units outside $S$

$J, C, J_{S}, C_{S}, U$ : Idele group of $\Omega$, i.e., the union of $J_{K}$ when $K$ runs over the finite subextension of $\Omega$, idele class group of $\Omega, \ldots \ldots$

$A^{\prime}=\operatorname{Hom}\left(A, \Omega_{\Im}{ }^{\times}\right)$(in the local case, the same notation for $\operatorname{Hom}\left(A, \bar{k}_{P}{ }^{\times}\right)$)

$A^{*}=\operatorname{Hom}(A, \boldsymbol{R} / \boldsymbol{Z}):$ Pontrjagin dual group of $A$

We prove following theorems.

THEOREM 1. If $M$ is a finitely generated ${ }^{2)} G$-module, then it holds (topological) isomorphisms

$$
\hat{H}^{i}\left(\operatorname{Hom}\left(M, C_{s}\right)\right) \cong \hat{H}^{2-i}(M)^{*}, \quad i \leqq 0
$$

As $M$ is finitely generated, there exists an exact sequence

$$
0 \longrightarrow F_{1} \longrightarrow F_{0} \longrightarrow M \longrightarrow 0
$$

with finitely generated torsion-free ${ }^{2)} G$-modules $F_{0}, F_{1}$. We can construct an exact sequence

$$
\begin{gathered}
\longrightarrow \hat{H}^{i-1}\left(\operatorname{Hom}\left(F_{0}, C_{S}\right)\right) \longrightarrow \hat{H}^{i-1}\left(\operatorname{Hom}\left(F_{1}, C_{S}\right)\right) \stackrel{\boldsymbol{\varphi}_{i}}{\longrightarrow} \hat{H}^{i}\left(\operatorname{Hom}\left(M, C_{S}\right)\right) \\
\longrightarrow \hat{H}^{i}\left(\operatorname{Hom}\left(F_{0}, C_{S}\right)\right) \longrightarrow \hat{H}^{i}\left(\operatorname{Hom}\left(F_{1}, C_{S}\right)\right), i \leqq 0 .
\end{gathered}
$$

Duality theorem for torsion-free modules in the cohomology theory of finite groups says

$$
\hat{H}^{i}(g, \operatorname{Hom}(F, \bar{C})) \cong \hat{H}^{2-i}(g, F)^{*}
$$

if $g$ is a finite group, $F$ is a torsion-free $g$-module and $\bar{C}$ satisfies the certain

2) "Finitely generated" and "torsion-free" are as additive groups, i. e., as $Z$-modules, 
cohomological condition. We can take the limits and we have (topological) isomorphisms

$$
\hat{H}^{i}\left(\operatorname{Hom}\left(F, C_{S}\right)\right) \cong \hat{H}^{2-i}(F)^{*}, i \leqq 0
$$

The theorem concludes combining these and applying five lemma.

REMARK. As Hom $\left(, C_{S}\right)$ is not an exact functor, we can not prove duality in other dimensions. Compare with the local case ([6]. Section 2).

Now we consider natural homomorphisms

$$
\begin{aligned}
& f: \hat{H}^{1}\left(M^{\prime}\right) \longrightarrow \prod_{P \in S} \hat{H}^{1}\left(k_{P}, M^{\prime}\right), \\
& g: \hat{H}^{2}(M) \longrightarrow \prod_{P \in S} H^{2}\left(k_{P}, M\right) .
\end{aligned}
$$

We denote the kernels of them by $\operatorname{Ker}^{1}\left(M^{\prime}\right)$ and $\operatorname{Ker}^{2}(M)$ respectively.

THEOREM 2. If the order of the torsion part of $M$ is a unit in $k_{S}$, there exists duality between $\operatorname{Ker}^{1}\left(M^{\prime}\right)$ and $\operatorname{Ker}^{2}(M)$ and they are finite modules.

There exists an exact sequence

$$
\operatorname{Hom}_{G}\left(M, J_{S}\right) \longrightarrow \operatorname{Hom}_{G}\left(M, C_{S}\right) \stackrel{\delta}{\longrightarrow} H^{1}\left(M^{\prime}\right) \stackrel{f}{\longrightarrow} \prod_{P \in S} \hat{H}^{1}\left(k_{P}, M^{\prime}\right)
$$

induced from

$$
0 \longrightarrow M^{\prime} \longrightarrow \operatorname{Hom}\left(M, J_{S}\right) \longrightarrow \operatorname{Hom}\left(M, C_{S}\right) \longrightarrow 0
$$

The map $\delta$ is continuous and the universal norms of $\operatorname{Hom}\left(M, C_{s}\right)$ maps to zero. So we have a commutative diagram

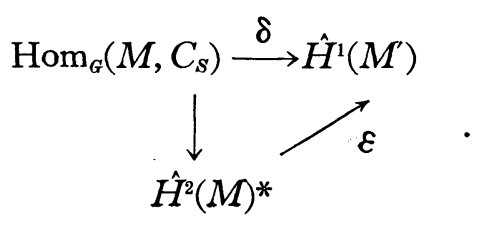


Then the theorem follows from the exact sequence

$$
\prod_{P \in S} \hat{H}^{2}\left(k_{P}, M\right)^{*} \stackrel{\eta}{\longrightarrow} \hat{H}^{2}(M)^{*} \stackrel{\varepsilon}{\longrightarrow} \hat{H}^{1}\left(M^{\prime}\right) \stackrel{f}{\longrightarrow} \prod_{P \in S} \hat{H}^{1}\left(k_{P}, M^{\prime}\right) .
$$

where II means the restricted direct product consisting of the elements whose components vanish the images of $\hat{H}^{2}\left(o_{P}, M\right)$ for almost all $P$.

REMARK. If $\operatorname{scd}_{l} G=2$ for some prime $l, \hat{H}^{0}\left(\operatorname{Hom}\left(M, C_{S}\right)\right) \rightarrow \hat{H}^{\circ}\left(\operatorname{Hom}\left(F_{0}, C_{S}\right)\right)$ is injective at $l$-primary part by Theorem 1 and the sequence (2). Especially in the case

$$
0 \longrightarrow \boldsymbol{Z} \stackrel{m}{\longrightarrow} \boldsymbol{Z} \longrightarrow \boldsymbol{Z} / m \boldsymbol{Z} \longrightarrow 0, m=l^{e},
$$

this means (in number field case) that

$$
C_{S, m}{ }^{G} \cap N_{G} C_{S}=N_{G} C_{S, m}
$$

If $M$ is finite and its order is a unit in $k_{S}$, surjectivity of $N_{G} \operatorname{Hom}\left(M, J_{S}\right) \rightarrow$ $N_{G} \operatorname{Hom}\left(M, C_{S}\right)$ follows from the fact that $N_{G} \operatorname{Hom}\left(M, C_{S}\right)=N_{G / H} N_{H} \operatorname{Hom}\left(M, C_{S}\right)$ and $\operatorname{Hom}_{G}\left(M, J_{S}\right) \rightarrow \operatorname{Hom}_{G}\left(M, C_{S}\right)$ has finite kernel. As $C_{S}=C / U$ (see the last part of section 2), $N_{G} C_{S}=D_{k} U_{k} / U_{k}$ holds, where $D_{k}$ is the set of the usual universal norms of the idele classes. Then the above equation means that the $l$-primary torsion part of $D_{k} U_{k} / U_{k}$ comes from the universal norms of $J_{S, m}$, or from the archimedean parts. If $S=$ all the primes in $k$, this is the case $\left(U_{k}=1\right)$ and $\operatorname{scd} G=2$. But is it true in general ?

2. Proof of Theorem 1. Let $A$ be a $G$-module such that every $A^{H}$ has compact topology and $G$ operates continuously on $A^{H}$ for any open normal subgroup $H$. Then $\hat{H}^{i}(G, A), i \leqq 0$, has compact topology because each $\hat{H}^{i}\left(G / H, A^{H}\right)$ has compact topology. (When $i>0$ we consider as $\hat{H}^{i}(G, A)$ has discrete topology for any $G$-module $A$.) As $\lim$ is an exact functor for compact modules, it holds

$$
\hat{H}^{i}(G, A)=\lim Z^{i}\left(G / H, A^{H}\right) / \lim B^{i}\left(G / H, A^{H}\right), \quad i \leqq 0,
$$

where $Z^{i}, B^{i}$ are cocycles and coboundaries respectively. As the cohomological deflation $\hat{H}^{i}\left(G / H^{\prime}, A^{H^{\prime}}\right) \rightarrow \hat{H}^{i}\left(G / H, A^{H}\right)$ maps $Z^{i}\left(G / H^{\prime}, A^{H^{\prime}}\right)$ into $Z^{i}(G / H$, $\left.N_{H / H^{\prime}} A^{H^{\prime}}\right)$ for $i<0$, every element of $\lim Z^{i}\left(G / H, A^{H}\right)$ has an $H$-component contained in $N_{H} A$. Therefore $\lim Z^{i}\left(G \overleftarrow{/ H}, A^{H}\right)=\lim Z^{i}\left(G / H, E_{H}\right)$ for any closed 
submodule $E_{H}$ of $A^{H}$ containing $N_{H} A$ and satisfying $N_{H / H^{\prime}} E_{H^{\prime}} \subset E_{H}$. The same is true for $B^{i}$ because coboundary maps commute with $\underset{\longleftarrow}{\longleftarrow}$, so

$$
\hat{H}^{i}(G, A)=\lim \hat{H}^{i}\left(G / H, E_{H}\right), \quad i<0 .
$$

Especially

$$
\begin{aligned}
& \hat{H}^{0}(G, A)=A^{G} / N_{G} A, \\
& \hat{H}^{-1}(G, A)=\left(\lim ^{\operatorname{Ker}} N_{G / H}\right) /\left(\lim ^{\longleftarrow} I A^{E}\right)
\end{aligned}
$$

hold. In the above $I A^{H}$ is generated by $(\sigma-1) a, \sigma \in G, a \in A^{H}$. Let $\bar{C}$ be a $G$ module satisfying the above conditions, and $M$ be a finitely generated $G$ module. Then $\operatorname{Hom}\left(M, \overline{C^{H}}\right)$ (which is equal to $\operatorname{Hom}_{H}(M, \bar{C})$ if $H$ is sufficiently small) has a topology induced from that of $\overline{C^{H}}$ and we can apply the above remark to $\operatorname{Hom}(M, \bar{C})$. When $N_{H} \bar{C}$ is divisible for any $H$, we can construct an exact sequence (2) replaced $C_{S}$ by $\bar{C}$. First we consider the case $i=0$. Homomorphism $\varphi_{0}: \hat{H}^{-1}\left(\operatorname{Hom}\left(F_{1}, \bar{C}\right)\right) \rightarrow \hat{H}^{0}(\operatorname{Hom}(M, \bar{C}))$ is given as follows. Let $\left(\alpha_{H}\right)$ $\in \hat{H}^{-1}\left(\operatorname{Hom}\left(F_{1}, \bar{C}\right)\right)$. Then we may assume $\alpha_{H} \in N_{H} \operatorname{Hom}\left(F_{1}, \bar{C}\right)$. If $H$ operates trivially on $F_{0}, F_{1}$, then $N_{H} \operatorname{Hom}\left(F_{1}, \bar{C}\right)=\operatorname{Hom}\left(F_{1}, N_{H} C\right)$. As $N_{H} \bar{C}$ is divisible, $\alpha_{H}$ is an image of $\beta_{H} \in \operatorname{Hom}\left(F_{0}, N_{H} \bar{C}\right)=N_{H} \operatorname{Hom}\left(F_{0}, \bar{C}\right)$. Then we define $\varphi_{0}\left(\left(\alpha_{H}\right)\right)=\left(N_{G / H}\left(\beta_{H}\right)\right)$ $\epsilon \hat{H}^{\circ}(\operatorname{Hom}(M, \bar{C}))$. Other homomorphisms are natural ones, and the proof of exactness is easy. When $i<0$, we take $\operatorname{Hom}\left(M, N_{H} \bar{C}\right)$ for $E_{H}$ in (11). (We only consider open subgroups $H$ such that $M^{H}=M$. This restriction does not affect to projective limits). As $N_{H} \bar{C}$ is divisible,

$$
0 \rightarrow \operatorname{Hom}\left(M, N_{H} \bar{C}\right) \rightarrow \operatorname{Hom}\left(F_{0}, N_{H} \bar{C}\right) \rightarrow \operatorname{Hom}\left(F_{1}, N_{H} \bar{C}\right) \rightarrow 0
$$

is exact. So we have an exact sequence

$$
\begin{aligned}
& \rightarrow \hat{H}^{i-1}\left(G / H, \operatorname{Hom}\left(F_{0}, N_{H} \bar{C}\right)\right) \rightarrow \hat{H}^{i-1}\left(G / H, \operatorname{Hom}\left(F_{1}, N_{H} \bar{C}\right)\right) \rightarrow \\
& \hat{H}^{i}\left(G / H, \operatorname{Hom}\left(M, N_{H} \bar{C}\right)\right) \rightarrow \hat{H}^{i}\left(G / H, \operatorname{Hom}\left(F_{0}, N_{H} \bar{C}\right)\right) \rightarrow \hat{H}^{i}\left(G / H, \operatorname{Hom}\left(F_{1}, N_{H} \bar{C}\right)\right) .
\end{aligned}
$$

Every term has compact topology and every map is continuous. So projective limits keep the exactness and we have (2) by considering (11).

Cup product gives a natural map

$$
\hat{H}^{i}\left(G / H, \operatorname{Hom}_{H}(M, \bar{C})\right) \rightarrow \operatorname{Hom}\left(\hat{H}^{j}\left(G / H, M^{B}\right), \hat{H}^{i+j}\left(G / H, \overline{C^{H}}\right)\right),
$$


We consider the case that $\bar{C}$ is a class formation and $i+j=2$. Then we have

$$
\hat{H}^{i}\left(G / H, \operatorname{Hom}_{H}(M, \bar{C})\right) \rightarrow \hat{H}^{2-i}\left(G / H, M^{H}\right)^{*} .
$$

If $i \leqq 0$ or $i \geqq 2$, we can take the limits, and we have a continuous map

$$
\hat{H}^{i}(G, \operatorname{Hom}(M, \bar{C})) \rightarrow \hat{H}^{2-i}(G, M)^{*}, i \neq 1 .
$$

LEMMA [4. Chap. 4, Theorem 14]. If $F$ is a finitely generated torsion-free $G$-module and $C$ is a class formation,

$$
\hat{H}^{i}(G, \operatorname{Hom}(F, C)) \rightarrow \hat{H}^{2-i}(G, F)^{*}
$$

is an isomorphism for any integer $i$.

As $\hat{H}^{1}(G, F)$ is finite, Lemma is valid also for $i=1$. Now we can prove Theorem 1 replaced $C_{S}$ by $\bar{C}$.

Proposition. Let $M$ be a finitely generated G-module. Let $\bar{C}$ be a class formation such that every $\overline{C^{H}}$ is compact and every $N_{H} \bar{C}$ is divisible. Then

$$
\hat{H}^{i}(\operatorname{Hom}(M, \bar{C})) \rightarrow \hat{H}^{2-i}(M)^{*}, i \leqq 0,
$$

are isomorphisms.

PROOF. We have a commutative diagram

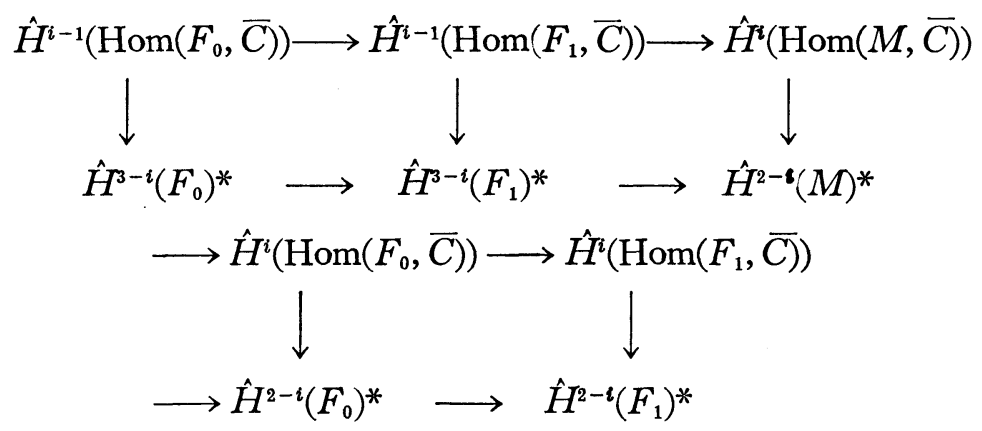

with exact rows. All the vertical arrows except the middle one are isomorphisms by the lemma. Therefore Proposition follows from Five Lemma. 
Now we prove Theorem 1 . We first prove that $C_{S}$ is a class formation. In fact $C_{S}$ is isomorphic to $C / U$ which is a class formation because $U$ is cohomologically trivial. There exists a natural monomorphism $C_{S, K} \rightarrow C_{K} / U_{K}$ considering $J_{S, K}$ as a subgroup of $J_{K}$. Cokernel of this monomorphism is finite by finiteness of class number, and $C_{K} / U_{K}$ is contained in the image of some $C_{S, L}$. For we take $L$ as a class field corresponding to $J_{s, K} U_{K} K / K$, then the group theoretical Principal Ideal Theorem [1.Chap.13,4] shows that $C_{K} \subset J_{S, L} U_{L} L / L$. Therefore $C_{S}$ is isomorphic to $C / U$, so we can replace $C_{S}$ by $C / U$ in Theorem 1 . In the number field case, we replace $C / U$ by $C_{0} / U$ where $C_{0}$ is a subgroup of $C$ consisting of the elements of volume 1 . As $C^{H} / C_{0}{ }^{H}$ is uniquely divisible, this rep'acement causes no change to cohomology groups. $C_{0}{ }^{H}$ is compact and the universal norms of $C_{0}$ are $D_{0} U$ where $D_{0}$ is the usual universal norms in $C_{0}$. So $C_{0} / U$ satisfies the conditions of Proposition. In the function field case, we replace $C^{H}$ by $\hat{C}^{H}$, where $\hat{C}^{H}$ is such that $\hat{C}^{H} / U^{H} \cong \hat{Z}$ is a compactification of $\boldsymbol{Z}$ by the ideal topology. This replacement also causes no change to cohomology. As $\hat{C} / U$ has trivial universal norms, it satisfies the conditions of Proposition. This completes the proof of Theorem 1.

3. Proof of Theorem 2. Exact sequence

$$
0 \longrightarrow \Omega_{S}{ }^{\times} \longrightarrow J_{S} \longrightarrow C_{S} \longrightarrow 0
$$

induces

$$
0 \longrightarrow M^{\prime} \longrightarrow \operatorname{Hom}\left(M, J_{S}\right) \longrightarrow \operatorname{Hom}\left(M, C_{S}\right) \longrightarrow 0
$$

which is exact by the assumption for the torsion part of $M$. Then

$$
\operatorname{Hom}_{G}\left(M, J_{S}\right) \longrightarrow \operatorname{Hom}_{G}\left(M, C_{S}\right) \stackrel{\delta}{\longrightarrow} \hat{H}^{1}\left(M^{\prime}\right) \longrightarrow \hat{H}^{1}\left(\operatorname{Hom}\left(M, J_{S}\right)\right)
$$

is exact. As there is a natural injection $\hat{H}^{1}\left(\operatorname{Hom}\left(M, J_{S}\right)\right) \rightarrow \Pi \hat{H}^{1}\left(k_{P}, M^{\prime}\right)$, we have the exact sequence (7). We first prove finiteness $\operatorname{of} \operatorname{Ker}^{1}\left(M^{\prime}\right)$. This is equivalent to finiteness of cokernel of $\operatorname{Hom}_{G}\left(M, J_{S}\right) \rightarrow \operatorname{Hom}_{G}\left(M, C_{S}\right)$. We have seen in section 2 that $C_{S}{ }^{H}$ is contained in some $C_{S, K}$. So we have $\operatorname{Hom}_{G}\left(M, J_{S}\right)=\operatorname{Hom}_{G}$ $\left(M, J_{S, K}\right), \operatorname{Hom}_{G}\left(M, C_{S}\right)=\operatorname{Hom}_{\tilde{f}}\left(M, C_{S, K}\right)$ for some finite extension $K$. Then replacing $H$ by smaller one if necessary, we have a commutative diagram with exact rows

$$
\begin{aligned}
\operatorname{Hom}_{G}\left(M, J_{S}\right) & \longrightarrow \operatorname{Hom}_{G}\left(M, C_{S}\right) \longrightarrow H^{1}\left(M^{\prime}\right) \\
\| & \vdots \\
\operatorname{Hom}_{G / H}\left(M, J_{S, K}\right) & \longrightarrow \operatorname{Hom}_{G / H}\left(M, C_{S, K}\right) \longrightarrow \operatorname{Ext}_{G / H}{ }_{G / H}\left(M, K_{S}^{\times}\right) .
\end{aligned}
$$


As $\hat{H}^{1}\left(M^{\prime}\right)$ is of torsion and $\operatorname{Ext}^{1}{ }_{G / H}\left(M, K_{S}^{\times}\right)$is finitely generated, cokernel we now concern is finite.

Next we prove the existence of an exact sequence (10) in the special cases $M=\boldsymbol{Z}$ or $\mu_{m}$ (the $m$-th roots of unity). When $M=Z, \hat{H}^{2}(\boldsymbol{Z})^{*} \cong \hat{H}^{1}(\boldsymbol{Q} / \boldsymbol{Z})^{*}$ $\cong G / G^{\prime}$. So we have a natural epimorphism

$$
\varepsilon^{\prime}: \hat{H}^{2}(\boldsymbol{Z})^{*} \cong G / G^{\prime} \rightarrow C l_{S} \cong \hat{H}^{1}\left(\Omega_{S}{ }^{\times}\right),
$$

where $C l_{S}$ is a factor group of the ideal (or divisor) class group in $k$ by the subgroup generated by primes in $S$ [2, Proposition 2.3]. The image of $\Pi \hat{H}^{2}\left(k_{P}, \boldsymbol{Z}\right)^{*}$ $\rightarrow H^{2}(\hat{\boldsymbol{Z}})^{*}$ is generated by the decomposition groups $G_{P}, P \in S$, so it is dense in the kernel of $\delta$. It is closed if $S$ is finite and then it is open as it is of finite index. So the image is always open and (10) is exact. When $M=\mu_{m}$, we have

$$
0 \longrightarrow \mu_{m} \longrightarrow \Omega_{S} \times \stackrel{m}{\longrightarrow} \Omega_{S} \times \longrightarrow \quad \text { (exact). }
$$

So

$$
\hat{H}^{1}\left(\Omega_{S}{ }^{\times}\right) \stackrel{m}{\longrightarrow} \hat{H}^{1}\left(\Omega_{S}{ }^{\times}\right) \longrightarrow \hat{H}^{2}\left(\mu_{m}\right)
$$

is exact. As $\hat{H}^{1}\left(\Omega_{S}{ }^{\times}\right)=C l_{S}$,

$$
\hat{H}^{2}\left(\mu_{m}\right)^{*} \longrightarrow \operatorname{Hom}\left(C l_{S}, \boldsymbol{Z} / m \boldsymbol{Z}\right)
$$

follows. By the natural epimorphism $G \rightarrow C_{S}$, we have

$$
0 \longrightarrow \operatorname{Hom}(C l, \boldsymbol{Z} / m \boldsymbol{Z}) \longrightarrow \operatorname{Hom}_{\text {cont }}(G, \boldsymbol{Z} / m \boldsymbol{Z})=\hat{H}^{1}(\boldsymbol{Z} / m \boldsymbol{Z}) .
$$

Combining two homomorphisms, we have

$$
\varepsilon^{\prime}: \hat{H}^{2}\left(\mu_{m}\right)^{*} \longrightarrow \hat{H}^{1}(\boldsymbol{Z} / m \boldsymbol{Z}) \text {. }
$$

The kernel of $\varepsilon^{\prime}$ is open by definition, and exactness of (10) follows from the relation between $\hat{H}^{2}\left(\Omega_{S}{ }^{\times}\right)$and $\hat{H}^{2}\left(k_{P}, \bar{k}_{P}{ }^{\times}\right)$[2, Corollary 2.9]. There is a natural $\operatorname{map} \operatorname{Hom}_{G}\left(M, J_{S}\right) \rightarrow \Pi \hat{H}^{2}(M)^{*}$ by the local duality [6, Theorem 1]. Then we have in cases $M=\boldsymbol{Z}$ or $\mu_{m}$

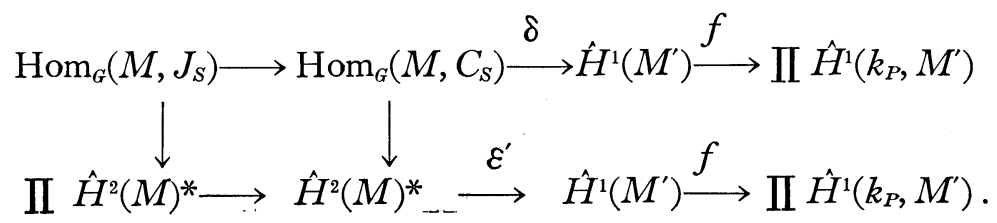


Square in the above diagram is commutative, so there exists a homomorphism $\operatorname{Im} \delta \rightarrow \operatorname{Im} \varepsilon^{\prime}$. This is an epimorphism, as the image of $\operatorname{Hom}_{G}\left(M, C_{S}\right)$ in $\hat{H}^{0}(\mathrm{Hom}$ $\left.\left(M, C_{S}\right)\right)=\hat{H}^{2}(M)^{*}$ is dense and the kernel of $\varepsilon^{\prime}$ is open. As $\operatorname{Im} \delta=\operatorname{Im} \varepsilon^{\prime}$ and they are finite, $\operatorname{Im} \delta \rightarrow \operatorname{Im} \varepsilon^{\prime}$ must be an isomorphism. Then the continuity of $\delta$ follows from that of $\varepsilon^{\prime}$ and $N_{G / H} \operatorname{Hom}\left(M, C_{S}{ }^{B}\right)$ goes to zero for some open subgroup $H$.

Now we consider the general case. Let $H$ be an open normal subgroup which operates trivially on $M$. As the diagram

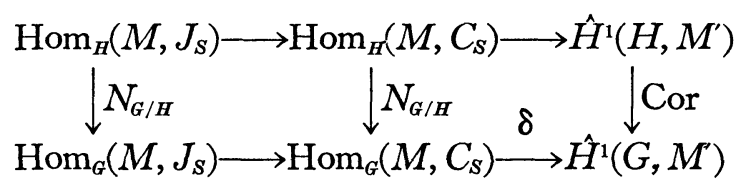

is commutative, $\delta$ maps $N_{G / H^{\prime}} \operatorname{Hom}\left(M, C_{S}{ }^{H^{\prime}}\right)=N_{G / H} N_{H / H^{\prime}} \operatorname{Hom}\left(M, C_{S}{ }^{H^{\prime}}\right)$ to zero for sufficiently small $H^{\prime}$. So $\delta$ is continuous and maps the universal norms $N_{G}$ Hom $\left(M, C_{S}\right)$ to zero. This is equivalent to the existence of a commutative diagram (9) with continuous map $\varepsilon$, because the image of $\operatorname{Hom}_{G}\left(M, C_{S}\right)$ is dense in $\hat{H}^{2}(M)^{*}$. Then we have a commutative diagram

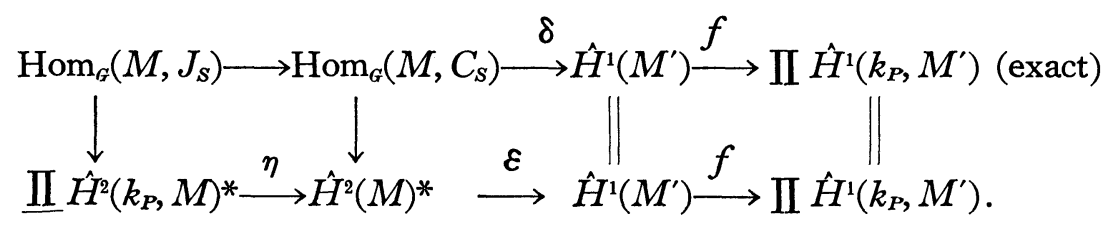

$\operatorname{Hom}_{G}\left(M, J_{Q}\right)$ is dense in $\underline{\Pi} \hat{H}^{2}\left(k_{P}, M\right)^{*}$ by the local duality, and the image of $\eta$ is open. So the bottom row is also exact. By

$$
0 \longrightarrow \operatorname{Ker}^{2}(M) \longrightarrow \hat{H}^{2}(M) \longrightarrow \mathrm{II} \hat{H}^{2}\left(k_{P}, M\right) \text { (exact) }
$$

and by the closedness of $\operatorname{Im} \eta$, the cokernel of $\eta\left(=\operatorname{Ker}^{1}\left(M^{\prime}\right)\right)$ is dual to $\operatorname{Ker}^{2}(M)$. This completes the proof of Theorem 2 .

\section{REFERENCES}

[1] E. ARTIN AND J. TAte, Class Field Theory.

[2] A. BRUMER, Galois groups of extenisons of algebraic number fields with given ramification, Michigan Math. J., 13(1966).

[ 3 ] K. HoechsmanN, Zum Einbettungsproblem, J. für Math., 229 (1968). 
[4] S. LANG, Rapport sur la cohomologie des groupes.

[ 5 ] G. PoITOU, Remarques sur l'homologie des groupes profinis, Colloques CNRS, 143(1966).

[6] T. TAKAHASHI, Galois cohomoloy of finitely generated modules, ibid.

[7] J. TATE, Duality theorems in Galois cohomology over number fields, Proc. Int. Congr. Stockholm, 1962.

MATHEMATICAL INSTITUTE

TÔHOKU UNIVERSITY

SENDAI, JAPAN 